\title{
„Die AO ist ein Sprungbrett und ein Hafen zugleich!“
}

Philip Wilbrandt

\author{
Am 11. und 12. Mai 2018 fand im Erfurter Augustinerkloster die 48. Jahrestagung \\ der AOTrauma Deutschland (AOTD) mit 105 Mitgliedern und 15 Gästen statt. Das \\ Erweiterte Präsidium informierte die Mitglieder über das gemeinsam Erreichte.
}

Insgesamt 104 wissenschaftliche Leiterinnen und Leiter organisierten 39 AO-Veranstaltungen in 2017. Die 16 Kurse waren zu 97,5\% ausgelastet mit insgesamt 1007 Teilnehmenden. Premiere hatten der mit der AOT Österreich und Schweiz gemeinsam ausgerichtete Masterkurs in Dresden (Leitung Bonaire/Kralinger/Platz u. Schaser) und der englischsprachige Masterkurs Hand in Düsseldorf (Linhart, Schäfer u. Windolf). Zu den 13 AOTD-Seminaren für Ärzte kamen 771 Teilnehmer/innen. Premiere hatte das Seminar in Kaiserslautern unter der Leitung von Alexander Hofmann als Teil des Seminarclusters Südwest (mit Trier/Junge und Koblenz/Gercek).

Erfreulicherweise findet in 2018 wieder eine deutschsprachige Master-Veranstaltung in Davos gemeinsam mit der AOT Österreich und Schweiz statt (mit Pol Rommens als Ko-Chairman aus Deutschland).

Für OP-Personal fanden in 2017 insgesamt sieben Kurse mit 370 und drei 1-tägige Seminare mit 118 Teilnehmenden statt. Das OP-Personal-Team begrüßte neue Mitglieder und betrachtet für die nähere Zukunft die Ausbildung von weiterem OP-Personal zur AO Faculty als Schwerpunktaufgabe.

Die Teilnahmegebühren wurden nach einer Marktanalyse angepasst. Für AOT-Mitglieder gibt es jetzt bei allen Veranstaltungen eine Ermäßigung in Höhe von 10\%. Für Nichtmitglieder bedeuten die neuen Preise eine leichte Erhöhung. Bei ORP-Veranstaltungen wurden die Gebühren gesenkt.

Die konsequente Umsetzung der Regel: „AO Faculty muss AOTrauma Mitglied sein“ wird mittlerweile durchweg akzeptiert. Die Weiterbildung der AO Faculty durch individuelles Coaching, das 2-mal jährlich stattfindende Faculty Education Program (FEP), das bisher von knapp 180 Ärzten absolviert wurde, sowie das im November 2018 zum 1. Mal in Deutschland durchgeführte Chairman Education Program (CEP) sichern die hohe Qualität der AOT-Kurse in Deutschland. Zwei Faculty Mitglieder aus Deutschland durften an dem Europäischen Faculty Seminar in Davos teilnehmen.

Für Studierende unterstützt die AOTD 3 spezielle Veranstaltungen in Kooperation mit dem Jungen Forum der DGOU auf der DGCh-Jahrestagung (20 Teilnehmende) und dem DKOU (100) sowie der VSOU-Tagung (40). Im September 2018 fand der 1. AOTD Studierenden-Kurs in Halle statt. Dieser wird 2019 in Düsseldorf wiederholt.

Die Nachwuchsförderung 2017 führte 2 öffentliche Calls durch. Von den 20 eingereichten Anträgen wurden 6 mit

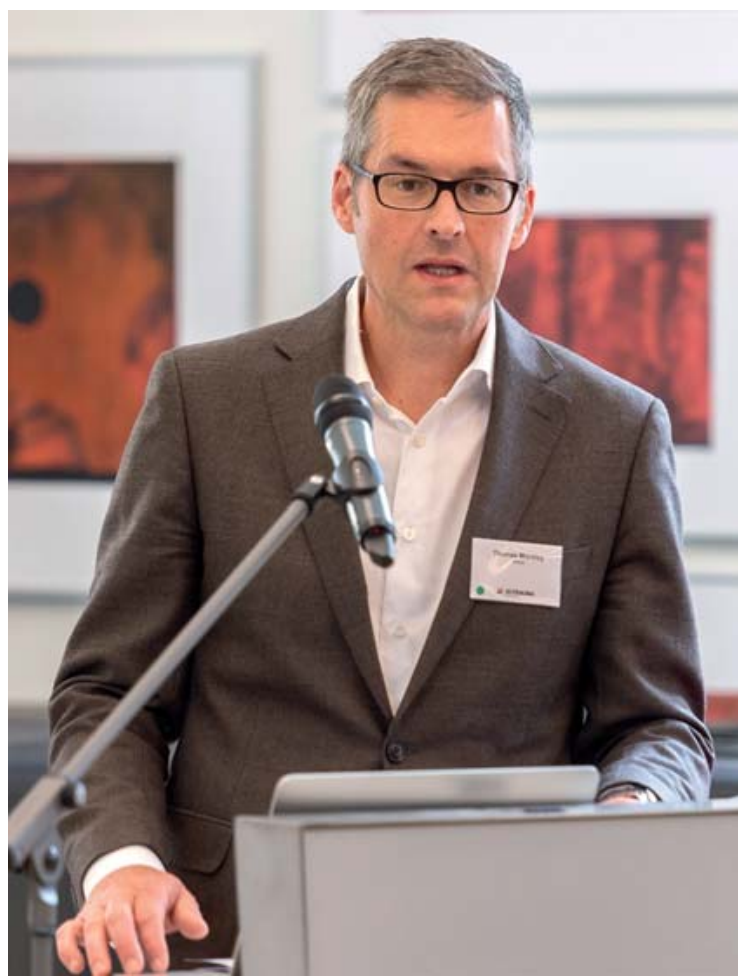

- Abb. 1 Gastgeber Thomas Mückley. Quelle: AO Foundation 


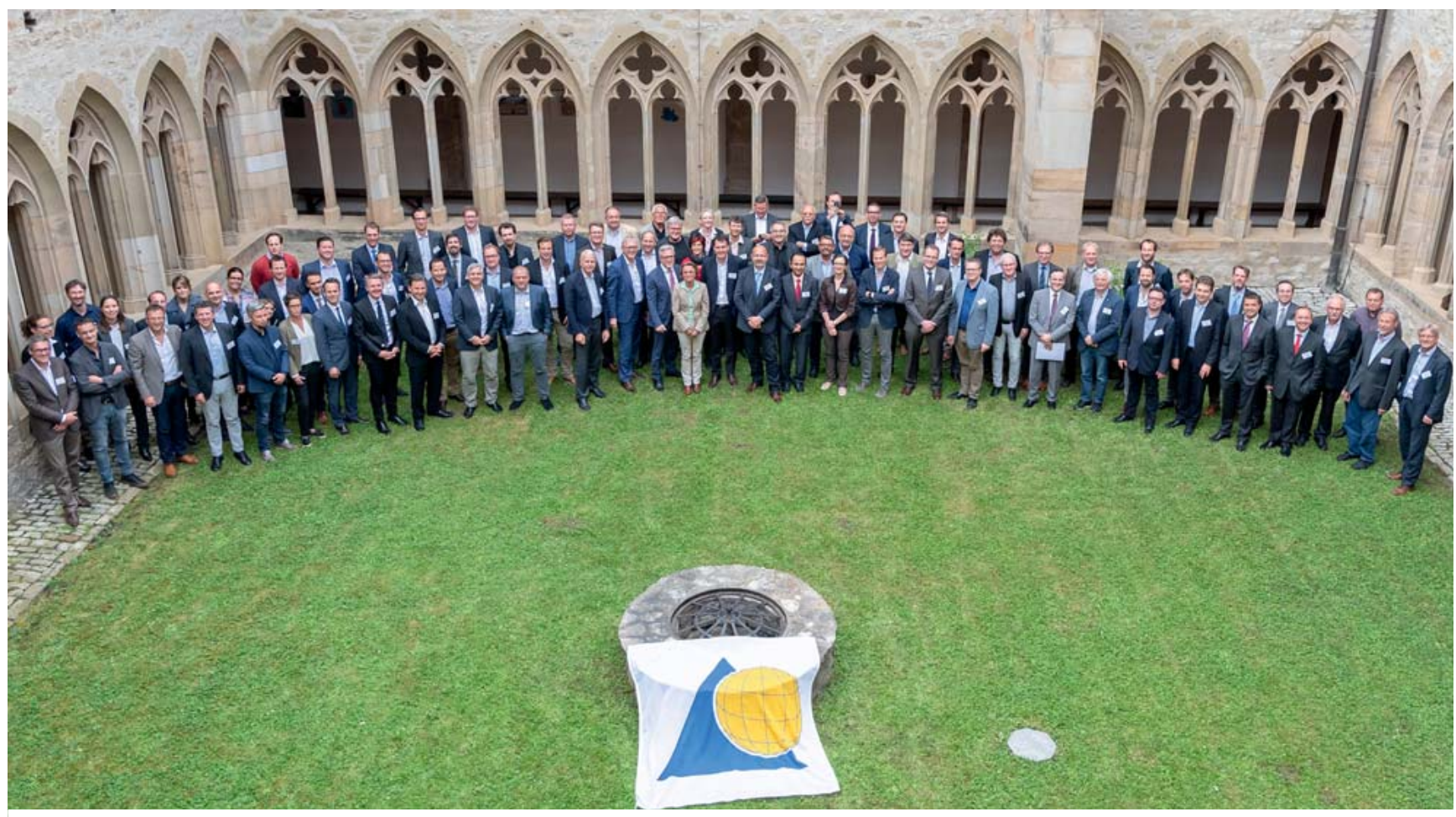

- Abb. 2 Gruppenphoto. Quelle: AO Foundation

einer Gesamtsumme von knapp 70000 EUR gefördert. Die Altersgrenze bei der Nachwuchsförderung wurde von 35 auf 40 Jahre angehoben und das Preisgeld auf max. 15000 EUR erhöht. Auf der Jahrestagung 2018 in Erfurt erfolgte die Auswahl von 2 Geförderten des 1 . Calls in 2018 durch eine geheime Abstimmung unter den anwesenden Mitgliedern.

Im Zuge des Mentoring-Angebotes fand auf dem DKOU 2017 das 1. AO Nachwuchsförderung Netzwerk-Treffen statt, was in 2018 wieder auf dem DKOU wiederholt wurde.

Zwei Ärzten wurde eine geförderte Teilnahme am AO Good Clinical Practice Course in Davos ermöglicht.

Der emotionale Höhepunkt der eh schon sehr stimmungsvollen Jahrestagung war zweifellos die Ernennung von Volker Bühren, Murnau, zum AOTD-Ehrenmitglied. In einer bewegenden Rede schilderte Ulrich Stöckle die Verdienste Volker Bührens für die Unfallchirurgie in Deutschland und ging dabei durchaus selbstkritisch auf die Beziehung zwischen der AO und Bühren ein. Minutenlange Standing Ovations würdigten Geehrten und Laudator.

Präsident Raschke dankte Gastgeber Thomas Mückley für die perfekte Ausrichtung der Tagung und die herzliche Gastfreundschaft. Er blickte zurück auf die nunmehr fast 6-jährige Amtszeit des Präsidiums. Die Position der AOT-

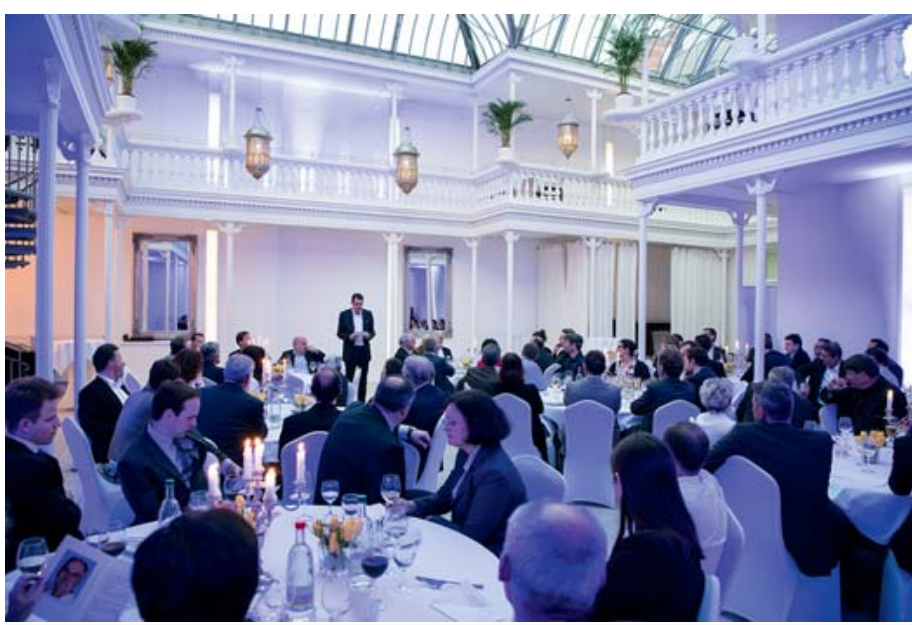

Abb. 3 Festabend. Quelle: AO Foundation

rauma Deutschland in der AOTrauma Welt wird über die Anzahl der Kurse (30\% aller AO-Veranstaltungen in Europa und 10\% weltweit), der Fellowship-Zentren (50\% aller in Europa und 25\% weltweit) und der Mitglieder (aktuell fast 750 , was $22 \%$ aller in Europa und $8 \%$ weltweit bedeutet) deutlich. Er bewertete als besonderes Highlight die Gründung der Akademie, die mit ihrer Diskussionskultur und Kameradschaft den AO-Gedanken im besten Sinne lebt und weiterreicht. Die gleichzeitige Öffnung der AO für jüngere Ärzte bietet eine organisatorische Plattform, um den Nachwuchs strukturierter in die 


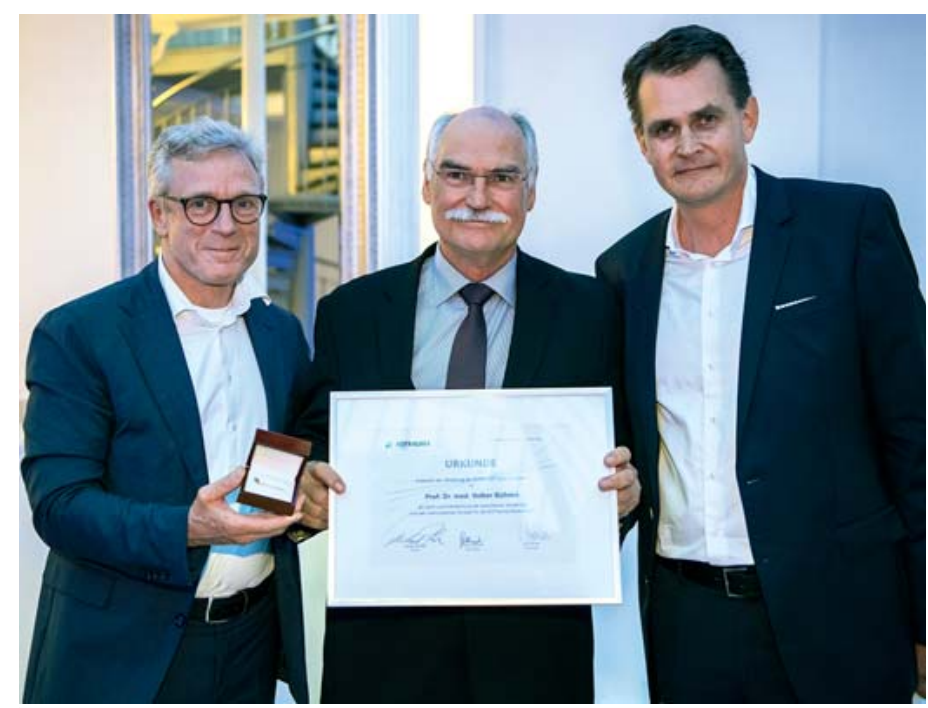

- Abb. 4 Raschke mit Ehrenmitglied Bühren und Stöckle. Quelle: AO Foundation

AO-Familie einzuführen und sie mit den ihnen anvertrauten Aufgaben wachsen zu lassen. Raschke dankte allen aktiven Mitgliedern im Namen des Präsidiums und drückte seinen Stolz auf das gemeinsam Erreichte aus, wobei für ihn nicht primär das Was, sondern das Wie den entscheidenden Unterschied ausmacht. Die Mitglieder der deutschen AO leben vorbildlich eine konstruktive Diskussionskultur. Sie behandeln sich mit Respekt, helfen sich gegenseitig mit Rat und Tat, und schätzen sich menschlich. Raschke schloss mit den Worten, dass für ihn „die deutsche AOTrauma ein Sprungbrett und ein Hafen zugleich ist".

Die nächste Jahrestagung ist die gemeinsame 3-LänderTagung mit der AOTrauma Österreich und Schweiz, die von Donnerstag, 9. Mai bis Samstag, 11. Mai 2019 in Hamburg stattfinden wird.

Philip Wilbrandt, Rechlin

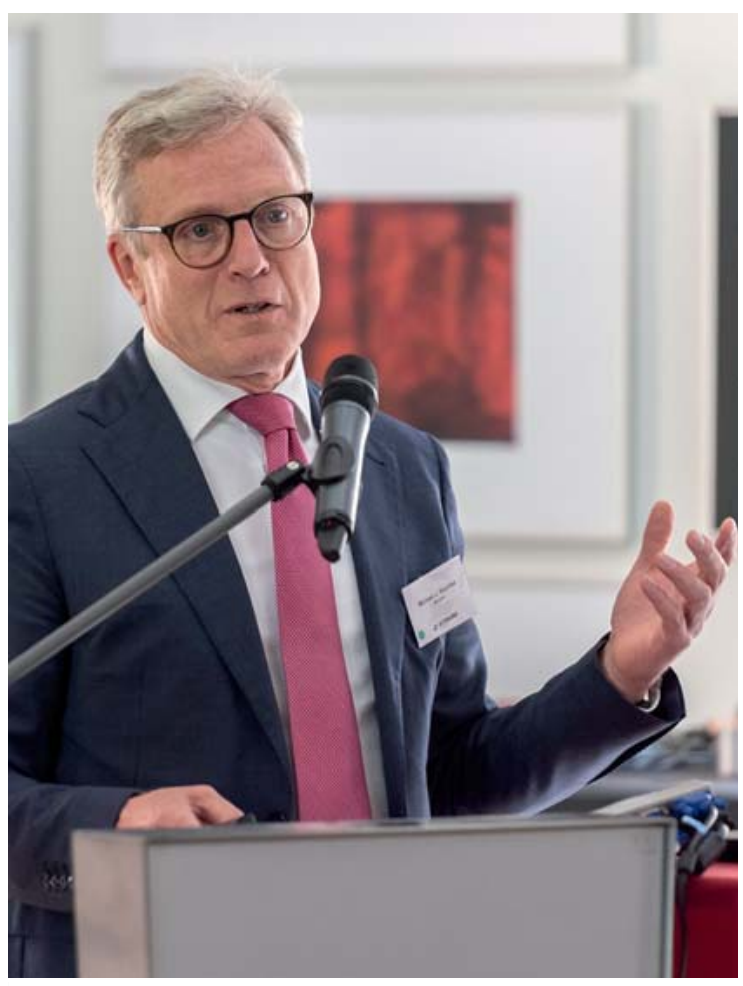

- Abb. 5 Präsident Michael Raschke. Quelle: AO Foundation

Bibliografie

DOI https://doi.org/10.1055/a-0750-6565

OP-JOURNAL 2018; 34: 334-336 @ Georg Thieme Verlag KG Stuttgart · New York ISSN 0178-1715 